\title{
Novel Robotic Approach for Minimally Invasive Aortic Heart Valve Surgery*
}

\author{
Izadyar Tamadon, Giorgio Soldani, Paolo Dario, Fellow, IEEE and Arianna Menciassi, Senior \\ Member, IEEE
}

\begin{abstract}
Aortic heart valve replacement is a major surgical intervention, traditionally requiring a large thoracotomy. However, current advances in Minimally Invasive Surgery and Surgical Robotics can offer the possibility to perform the intervention through a narrow mini thoracotomy. The presented surgical robot and proposed surgical scenario aims to provide a highly controllable means for efficiently conducting valve replacement by endoscopic vision. The robot, consisting of a series of joints, is a cable actuated manipulator for reaching the operative site and delivering the valve at the required position. The robot is equipped with endoscopic cameras (to find the hinge points) and three stabilizing flaps (to stabilize the manipulator) for guarantying the proper valve placement. The manipulator is validated by experimental results of flaps' force and camera visions in artificial vessels.
\end{abstract}

\section{INTRODUCTION}

Valvular heart disease is characterized by damage or defect of one of the four heart valves. Aortic Stenosis (AS narrowing of the aortic valve opening) is one of the most common valvular heart diseases among elderly people [1]. The AS prevalence in the elderly is around $12 \%$ and the prevalence of severe AS is $3.4 \%$ [2]. It occurs mainly in calcification or leaflet thickening, as a consequence of aging; but it may also be the result of different causes such as smoking, high cholesterol, high blood pressure and diabetes [3]. No pharmacologic treatment has been shown to attenuate the progressive of valve calcification, nor improve survival [4]. AS is a severe medical condition with an average survival of only 2 - 3 years [3]. Current treatment for AS includes Balloon Aortic Valvuloplasty (BAV) [5], Surgical Aortic Valve Replacement (SAVR) [6], [7], Transcatheter Aortic Valve Replacement (TAVR) [8] and Minimally Invasive Valve Surgery (MIVS) [9]. Currently, an estimated 300,000 replacement surgical procedures are carried out annually, worldwide. This figure is expected to grow up to 850,000 in 2050 [10]. However, precise positioning of the valve (including plane adjustment and rotation), paravalvular leakage, atrioventricular block with a need for pacemaker implantation in TAVR is controversial [11]. In general, the potential benefits of minimally invasive surgery are related to recovery time but still the gold standard in valve surgery [12].

*Research supported by Regione Toscana, Bando Fas Salute 2014, Valvetech.

Izadyar Tamadon (corresponding author), Paolo Dario and Arianna Menciassi are with the BioRobotics Institute, Scuola Superiore Sant'Anna, Pisa, 56025, ITALY (E-mail: \{i.tamadon, paolo.dario and arianna.menciassi\}@santannapisa.it).

Giorgio Soldani is with Consiglio Nazionale delle Ricerche, Istituto di Fisiologia Clinica (CNR), Laboratory for Biomaterials \& Graft Technology, Massa, 54100, ITALY. (E-mail: Giorgio.soldani@ifc.cnr.it).
With evolving robotics in MIS, there are further benefits in term of 3-dimensional visualization, tele-manipulation, motion scaling, and even smaller incisions [13], [14]. However, there are certain challenges before the widespread usage of this technique in heart surgery. The da Vinci robotic surgical system was the only FDA approved system for mitral valve surgery in 2002 and it was later approved for coronary revascularization in 2004 [15]. On the other hand, there has been a slow adoption of this technology in cardiac surgery for reasons related to procedural cost and lack of proper training [16]. As a response to the mentioned limitations, specific robotic systems dedicated to heart surgery have been introduced [17].

In the field of aortic heart valve deployment, visualization is also a major bottleneck. So, intraoperative MRI technology has been used to position aortic heart valve more precisely [18], [19]. 3D ultrasound guided motion compensation systems have also been reported for mitral valve delivery [20]. However, these task-specific robotic systems are not adaptable enough to perform MIS procedures for aortic valve replacement.

In this paper a novel robot for performing MIVS is proposed. The robot is in the form of a flexible manipulator guided by endoscopic vision. Small endoscopic cameras on the robot tip are used to help the surgeons for positioning the aortic valve accurately with respect to the hinge points. Additionally, three flaps in the tip of manipulator are used to keep the aorta open and stabilize the manipulator in the intervention site. In the next section the manipulator and its functions are detailed, including the description of the target surgical scenario. Flaps and cameras' vision, which are peculiar of our design in comparison with traditional delivery systems for valves [21] are characterized in section III.

\section{MATERIALS AND METHODS}

\section{A. Platform design overview}

In minimally invasive heart valve surgery, surgeons can replace the aortic valve through a small incision between the ribs. In order to reduce the invasiveness of the surgical procedure, instruments need to be maneuvered along tortuous and narrow paths. Flexible manipulators are ideal for accessing such areas of the patient's chest through a small entry point. The proposed robotic system is driven by cables running through the robot body and responsible for actuating the robot in space. The complete surgical robotic system consists of a flexible manipulator, a visualization aid (navigator) and a control unit, as in "Fig. 1". The flexible manipulator is attached to a rigid base that is held fixed by an arm. This arm (Martin's arm, MARINAMEDICAL, USA) is attached to the patient's bed and it holds the manipulator base 
fixed with respect to the patient. It can be positioned manually in order to set the working space of the flexible manipulator in the most adequate position. The arm can support an estimated weight of $4 \mathrm{~kg}$, providing 360 degrees rotating angle, and height adjustment with quick fixing mechanism to answer the surgeons' requirements.

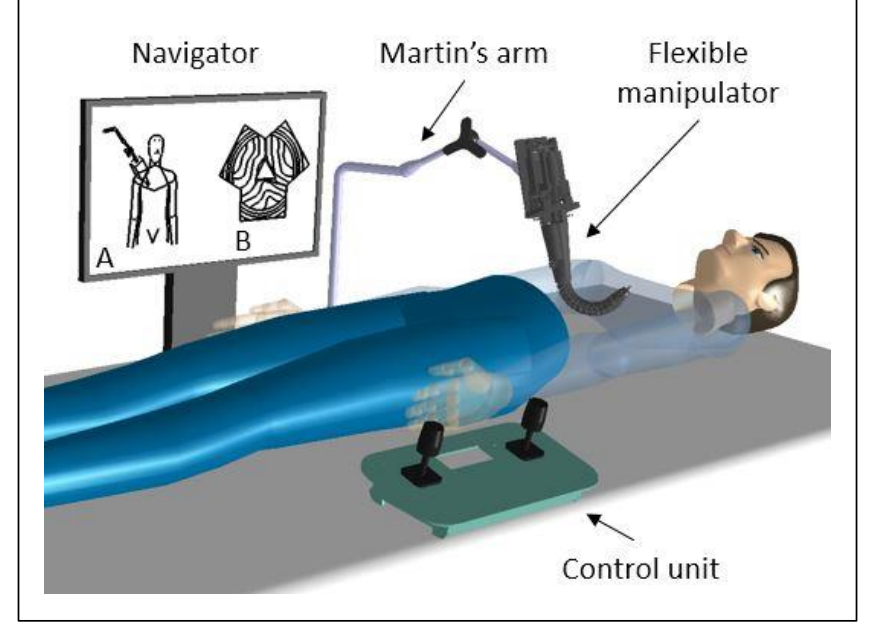

Figure 1. The proposed platform for aortic heart valve surgery. (The navigator: (A) Patient scans, (B) Endoscopic cameras vision).

Once the manipulator base is fixed, the flexible arm will be inserted in the patient's body through an incision in the intercostal region. The flexible manipulator has omnidirectional bending capabilities which are controlled by cables and servomotors. There are 4 cables arranged at 90 degrees intervals and running through the structure of the robot in dedicated cable channels. These cables are fixed to the tip of the robot at one end and the other end is reeled around a rotor which in turn is run by a servomotor (Series 2250 Faulhaber, Switzerland). Servomotors are attached to worm gears with $1 / 10$ ratio in order to lock cables in the work space. Thus the cables displacement resolution is $20 \times 10^{-5}$ $\mathrm{mm}$. The robotic manipulator has an external and internal diameter of $27 \mathrm{~mm}$ and $23 \mathrm{~mm}$, respectively. The manipulator links are made by a ProJet MJP3600 (3D systems, USA) 3D printer machine with VisiJet M3 crystal. The links are assembled together with pins to hinder undesired movements. The manipulator and work space are showed in "Fig. 2". The final design will be fabricated with medical grade titanium for in-vivo validation.

\section{B. Robot features}

\section{1) Endoscopic Vision}

The manipulator is designed to provide the surgeons with the possibility to reach and explore the surgical area, and replace the heart valve with the help of endoscopic vision. Three cameras are positioned in the head of the flexible manipulator in $120^{\circ}$ configurations. FISCam ${ }^{1}$ (FISBA, Switzerland) cameras have been selected because of their small diameter (less than $2 \mathrm{~mm}$ ) and $120^{\circ}$ field of view. The visual depth of field is up to $5 \mathrm{~cm}$. The cameras include LED fibers which bundle circularly to provide illumination in the aorta. The integrated illumination power is controlled through control box.

${ }^{1}$ http://www.fisba.com/expertise/expertise-components/fisba-fiscam

\section{2) Stabilizing Flaps}

The normal diameter of the ascending aorta is influenced by several factors, including body size, age, gender and blood pressure but usually is less than $40 \mathrm{~mm}$ [22]. To match this range of diameter at the distal end of the manipulator, 3 flaps are designed to keep the aorta open. They also stabilize the manipulator during the procedure. In fact, in the case of aortic valve replacement, flaps are necessary to enhance the view provided by the cameras and prevent the collapse of the aorta wall. Flaps are designed to operate without hindering the bending radius of whole manipulator. For this purpose, a flexible shaft passing through the manipulator is used to open/close the flaps. In the closed state, flaps help to protect the valve within the body of the manipulator. Once at the site, the flaps open and help the manipulator to stay in the middle of aorta which is one of the advantages of the robot in comparison to other manipulators [23].

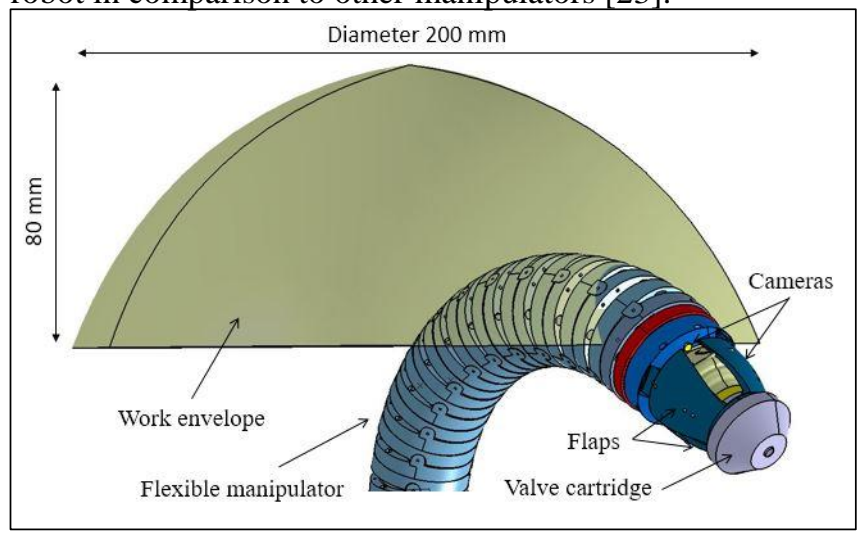

Figure 2. The flexible manipulator in maximum bent configuration with closed flaps and crimped artificial heart valve.

\section{3) Introducer and valve's cartridge}

The introducer keeps one of the most important roles of the whole system, i.e. valve releasing at the surgical site. It stays inside the flexible manipulator and can bend, thus it does not hamper the motion of the manipulator itself "Fig. 3". During the motion of the internal introducer, some motions in the external manipulator may occur. However these motions are damped due to the stabilizing effect of the flaps with vessels. The controlling commands are given by the surgeons through joysticks (integrated in the control unit), who are assisted by camera visions "Fig. 1". The introducer has 2 degrees of freedom not coupled with the flexible manipulator for accurate positioning of the valve inside the aorta. It provides translational movement of the valve to the release site and also a rotation about its axis for orientation of the valve. As the valve's frame and hinge points of the aorta should match each other, rotation is essential for proper placement. The distal end of the introducer has the valve cartridge attached with four magnets. The introducer can release the valve by a motor mounted on its body. Once the valve has been released in the aorta in the correct position and orientation, the introducer can be withdrawn by sliding internally in the manipulator.

The sutureless perceval aortic valve (Sorin Group, Saluggia, Italy) is used at many European cardiac surgery centers. Several reports evaluating implantation feasibility 
and valve safety in humans [24] are available. The Sorin's artificial valve can be crimped with a crimping device before being placed in the cartridge and introducer system.

The valve cartridge is designed to hold the crimped valve at the distal end of the introducer as in "Fig. 3". It is attached to the introducer with help of a magnetic base, as mentioned before. Once in position, the valve will emerge from the manipulator, and it will expand to its original size.

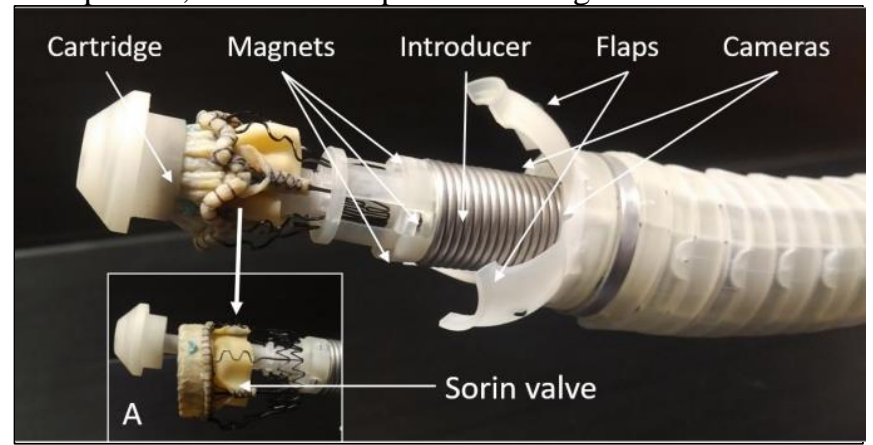

Figure 3. The introducer and valve cartridge. (A) Sorin valve while has been expanded.

\section{Surgery Protocol}

Heart valve replacement surgery with the proposed platform consists of two steps "Fig. 3".

\section{1) The preparation process}

Before the operation, the cartridge is prepared by surgeons using a crimping device. The cartridge is then attached to the introducer. After the attachment, the flaps of the manipulator are closed to secure the valve in the manipulator body. At this stage the manipulator is ready to be inserted in the patient's aorta. The base of the robot system is then moved into position, in line with the guidance from preoperative scans, to provide sufficient freedom for inserting the manipulator. This completes the preparation phase. The mini thoracotomy location can be defined preoperatively using patient specific surgical simulators [25]. The simulator allows moving a virtual replica of the manipulator in the patient virtual anatomy, in order to evaluate the best location of the mini thoracotomy.

\section{2) The delivery process}

After the first step, a preoperative simulation figures out the best approaching route to the aortic valve of the patient; based on this simulation, the delivery process may start (Cardiopulmonary bypass (CPB) is required). Flexibility and controllability of the manipulator help the surgeons' to find the calcified original valve and position the manipulator at an optimal pose. Usually this pose will be $3-4 \mathrm{~cm}$ away from the original calcified valve. At this point the flaps are expanded to stabilize the manipulator in aorta, widen the cameras' vision and lower the chance of vessel collapsing. The introducer is now able to rotate the valve and translate it to the expansion site. When the artificial heart valve is correctly aligned by the surgeon with the anatomical references (hinge points), the valve expansion process will start under the control unit guidance. After expansion, the introducer is retracted into the flexible manipulator, flaps will close and finally the flexible manipulator is ready to be retracted from patient's body.

\section{Experimental set-up}

In order to validate the performance of the manipulator and to study the manipulator stability in aorta, a simple experiment has been organized to measure the flaps force on the vessels. The experimental set-up is shown in "Fig. 4". The flap mechanism is actuated by a DC gearbox motor and internal gear ring via a flexible shaft. The flexible shaft, normally used for bicycles braking systems, is $23 \mathrm{~cm}$ in length. The flaps are connected to the internal gear ring by fishing cables, so they will open/close together. The force has been measured with Instron universal testing machine in different opening degrees. The results are shown in "Fig. 5". Also, In order to establish the feasibility of the visual system, the manipulator's tip was inserted to an artificial aortic model (Lifelikebiotissue, Canada). This examination is similar to finding the hinge points in aortic root.

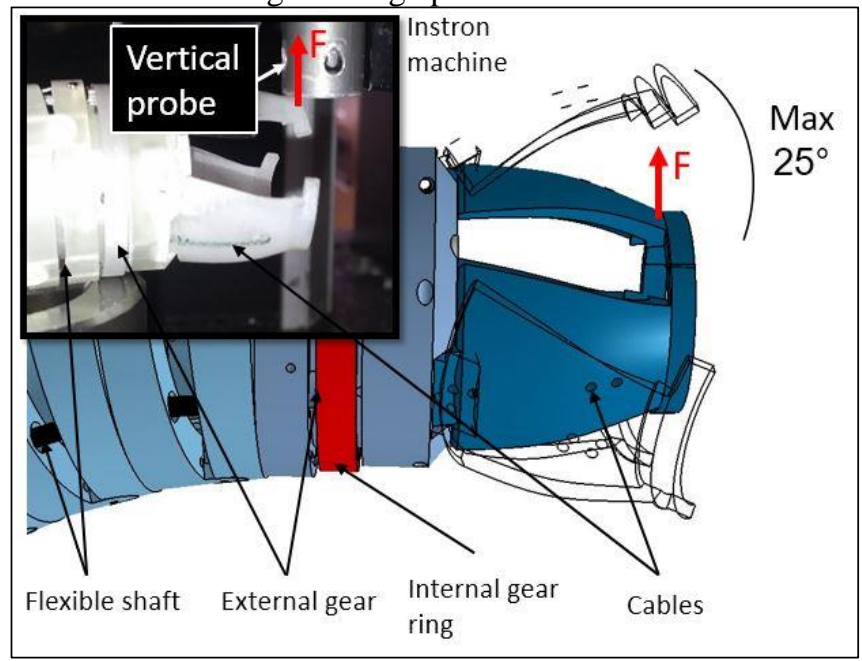

Figure 4. The experiment setup to measure openning force of flaps.

\section{RESULTS}

The flaps were commanded to open in different radiuses and the pushing force was recorded 5 times. The maximum force can be found in the closed position $(0.459 \mathrm{~N})$ and it decreases as the flap is opening to maximum radius.

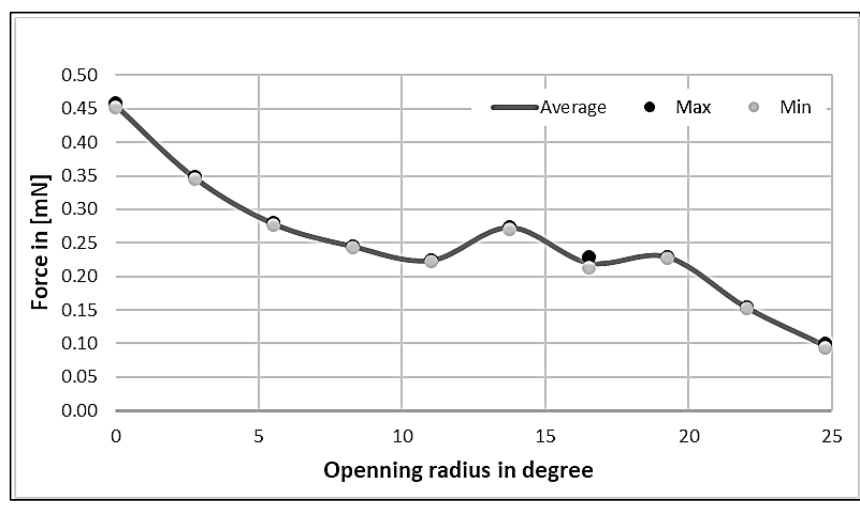

Figure 5. The Orthogonal force of one flap.

As can be seen in "Fig. 6", the camera can provide sufficiently clear views of the surgical site while the flaps 
are open, which can be considered as a proof of concept. The view of other cameras are not shown here but they are much similar to "Fig. 6" to find other hinge points.

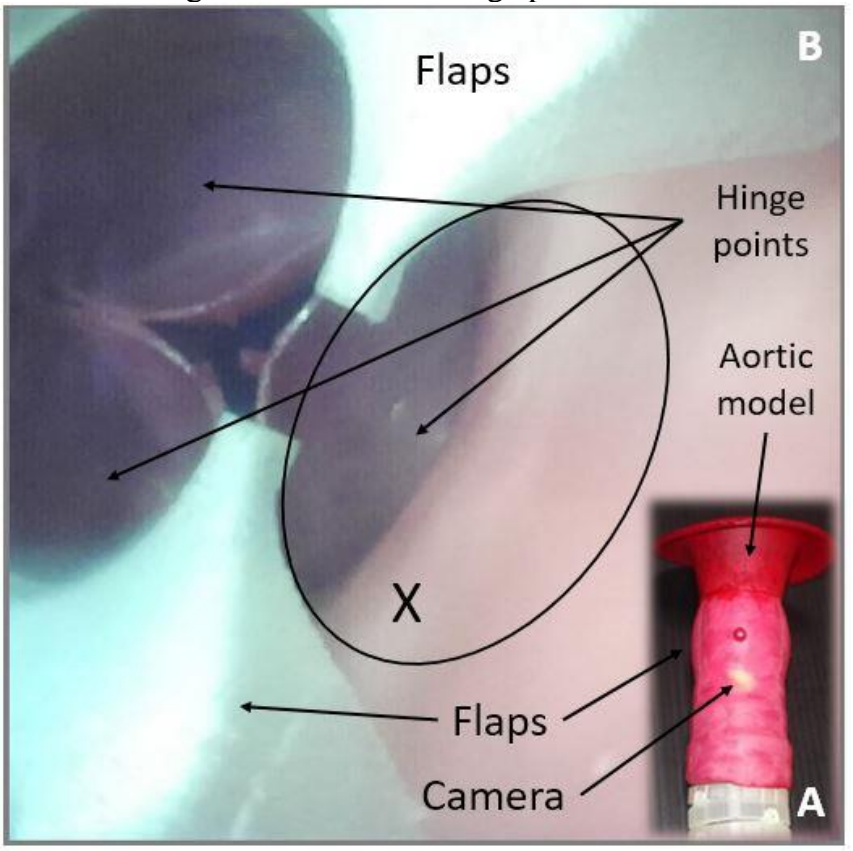

Figure 6. (A) Experimental set-up, (B) The view of one camera inside artificial vessel, (X) Expected region to find hinge points.

\section{CONCLUSION AND FUTURE WORKS}

A novel 4-DOF robotic system for MIVS with visual feedback is presented. MIVS involves bigger incisions in compare with TAVR, but it can deliver the valve more accurately. Experimental results indicate that the flaps even with small amount of force can stabilize the manipulator. Opening force is changing because of friction force of cables and tip of manipulator in different radiuses. Also, endoscopic cameras vision is sufficient to provide feedback for valve adjustment with rotations and translational movements provided by the robot. The robotic introducer is a proof of concept and is currently under development to improve efficacy for in-vivo tests. A more robust controller under external disturbances is also needed for controlling the manipulator in the sensitive MIS environment specifically, control strategies based on video tracking of the anatomical references with an automatic movement of the manipulator.

\section{REFERENCES}

M. A. Clark et al., "Clinical and economic outcomes after surgical aortic valve replacement in Medicare patients," Risk Manag. Healthc. Policy, vol. 5, p. 117, 2012.

[2] R. L. J. Osnabrugge et al., "Aortic stenosis in the elderly: Disease prevalence and number of candidates for transcatheter aortic valve replacement: A meta-analysis and modeling study," J. Am. Coll. Cardiol., vol. 62, no. 11, pp. 1002-1012, 2013.

[3] J. Joseph, S. Y. Naqvi, J. Giri, and S. Goldberg, "Aortic Stenosis: Pathophysiology, Diagnosis, and Therapy," Am. J. Med., vol. 130, no. 3, pp. 253-263, 2017.

[4] G. W. Eveborn, H. Schirmer, G. Heggelund, P. Lunde, and K. Rasmussen, "The evolving epidemiology of valvular aortic stenosis. the Tromsø study.," Heart, vol. 99, no. 6, pp. 396-400, 2013.
(BAV) as a bridge to aortic valve replacement in cancer patients who require urgent non-cardiac surgery.," Radiol. Oncol., vol. 48, no. 1, pp. 62-6, 2014.

[6] F. W. Mohr et al., "The German Aortic Valve Registry: 1-year results from 13680 patients with aortic valve disease," Eur. J. Cardio-thoracic Surg., vol. 46, no. 5, pp. 808-816, 2014.

[7] V. H. Thourani et al., "Contemporary real-world outcomes of surgical aortic valve replacement in 141,905 low-risk, intermediate-risk, and high-risk patients," Ann. Thorac. Surg., vol. 99, no. 1, pp. 55-61, 2015.

[8] M. J. Mack et al., "5-year outcomes of transcatheter aortic valve replacement or surgical aortic valve replacement for high surgical risk patients with aortic stenosis (PARTNER 1): A randomised controlled trial," Lancet, vol. 385, no. 9986, pp. 2477-2484, 2015.

[9] A. B. Goldstone and Y. Joseph Woo, "Minimally invasive surgical treatment of valvular heart disease," Semin. Thorac. Cardiovasc. Surg., vol. 26, no. 1, pp. 36-43, 2014.

[10] R. O. Bonow, M. B. Leon, D. Doshi, and N. Moat, "Management strategies and future challenges for aortic valve disease," Lancet, vol. 387, no. 10025, pp. 1312-1323, 2016.

[11] H. G. H. Thyregod et al., "Transcatheter versus surgical aortic valve replacement in patients with severe aortic valve stenosis: 1year results from the all-comers NOTION randomized clinical trial," J. Am. Coll. Cardiol., vol. 65, no. 20, pp. 2184-2194, 2015.

[12] T. Walther, J. Blumenstein, A. van Linden, and J. Kempfert, "Contemporary management of aortic stenosis: surgical aortic valve replacement remains the gold standard.," Heart, vol. 98 Suppl 4, p. iv23-9, 2012.

[13] R. C. Neely, M. Leacche, C. R. Byrne, A. V. Norman, and J. G. Byrne, "New approaches to cardiovascular surgery," Curr. Probl. Cardiol., vol. 39, no. 12, pp. 427-466, 2014.

[14] E. Rodriguez et al., "Pathway for surgeons and programs to establish and maintain a successful robot-assisted adult cardiac surgery program," J. Thorac. Cardiovasc. Surg., vol. 152, no. 1, pp. 9-13, 2016.

[15] W. Vernick and P. Atluri, "Robotic and minimally invasive cardiac surgery," Anesthesiol. Clin., vol. 31, no. 2, pp. 299-320, 2013.

[16] R. J. Shemin, "The future of cardiovascular surgery," Circulation, vol. 133, no. 25, pp. 2712-2715, 2016.

[17] A. Degani, H. Choset, A. Wolf, and M. A. Zenati, "Highly articulated robotic probe for minimally invasive surgery," Proc. IEEE Int. Conf. Robot. Autom., vol. 2006, pp. 4167-4172, 2006.

[18] E. Yeniaras et al., "Magnetic resonance based control of a robotic manipulator for interventions in the beating heart," 2011 IEEE Int. Conf. Robot. Autom., pp. 6270-6275, 2011.

[19] N. V. Navkar, Z. Deng, D. J. Shah, K. E. Bekris, and N. V. Tsekos, "Visual and force-feedback guidance for robot-assisted interventions in the beating heart with real-time MRI," Proc. IEEE Int. Conf. Robot. Autom., pp. 689-694, 2012.

[20] S. G. Yuen, N. V. Vasilyev, P. J. del Nido, and R. D. Howe, "Robotic tissue tracking for beating heart mitral valve surgery," Med. Image Anal., vol. 17, no. 8, pp. 1236-1242, 2013.

[21] "HEART VALVE TECHNOLOGY." [Online]. Available: https://www.dicardiology.com/channel/heart-valve-technology. [Accessed: 01-Feb-2018].

[22] R. Erbel and H. Eggebrecht, "Aortic dimensions and the risk of dissection," Heart, vol. 92, no. 1, pp. 137-142, 2006.

[23] G. P. Moustris, S. C. Hiridis, K. M. Deliparaschos, and K. M. Konstantinidis, "Evolution of autnomous and semi-autnomous robotic surgical systems: a review of the literature," Int. J. Med. Robot., vol. 7, no. April, pp. 375-392, 2011.

[24] S. Pfeiffer, T. Fischlein, and G. Santarpino, "Sutureless Sorin Perceval Aortic Valve Implantation," Semin. Thorac. Cardiovasc. Surg., vol. 29, no. 1, pp. 1-7, 2017.

[25] G. Turini et al., "Patient Specific Virtual and Physical Simulation Platform for Surgical Robot Movability Evaluation in SingleAccess Robot-Assisted Minimally-Invasive Cardiothoracic Surgery," 2017, pp. 211-220. 